\title{
REDESCRIPTION D'HELMINTHOXYS TIFLOPHILA et considérations sur la systématique des Oxyuridae parasites de Rongeurs.
}

\author{
J. P. HUGOT*
}

RÉSUMÉ. Redescription d'Helminthoxys tiflophila (Vigueras, 1943), parasite du Rongeur Capromys prehensilis (Caviomorpha). Analyse cladistique des relations phylogénétiques du genre Helminthoxys et des formes voisines : les Oxyuridae de Rongeurs et de Lagomorphes constituent un ensemble monophylétique. A l'intérieur de cet ensemble les parasites de Rongeurs Hystricognathes (Phiomorpha + Caviomorpha) s'opposent aux Oxyuridae parasites de Rongeurs Sciurognathes et de Lagomorphes.

\section{Redescription of Helminthoxys tiflophila and considerations about Systematic of Oxyuridae parasite in Rodents.}

SUMMARY. - Morphology - Helminthoxys tiflophila (Vigueras, 1943) parasite in Capromys prehensilis (Caviomorpha, Rodent) is redescribed. In the males of this species occurs a peculiar type of ventral cuticular swellings. The adherence with cuticule of females is not obtained by hooking, but by gluing : a ventral hypodermal secretion coagulates on surface of swellings.

- Systematic - The genus Helminthoxys Freitas, Lent and Almeida, 1937 is compared by means of cladistic method with the most closely related forms :

1 - The genera Helminthoxys and Octodonthoxys Quentin, Courtin and Fontecilla, 1975 cannot be distinguished using this method,

2 - Helminthoxys-Octodonthoxys is the sister group of Wellcomia, Sambon 1907, Protozoophaga Travassos, 1923.

3 - These four genera, all parasite in Hystricognathi Rodents may be opposed as a sister-group to the Oxyuridae parasite in Sciurognathi Rodents and Lagomorpha.

4 - The whole Oxyuridae parasite in Rodents (excepted Citellina Prendel, 1948) and Lagomorpha form a monophyletic group.

- Laboratoire de Zoologie (Vers), Muséum National d'Histoire Naturelle, 61 rue Buffon, F 75231 Paris Cedex 05.

Accepté le 22 novembre 1982. 


\section{Introduction}

Le genre Helminthoxys Lent, Freitas et Almeida, 1937 est parasite de nombreux Rongeurs Caviomorphes américains. L'étude d'H. tiflophila (Vigueras, 1943) parasite de Capromys prehensilis Poeppig révèle chez les mâles appartenant à cette espèce, un système d'accrochage ventral original, très différent de celui rencontré chez la plupart des autres oxyures parasites de Rongeurs. La position systématique du genre Helminthoxys est précisée.

\section{Morphologie}

- Matériel : 3 హెరิ et 3 우 adultes collectés dans le rectum d'un Capromys prehensilis capturé par V. Barus en 1968 dans la Sierra del Rosario (Provincia Pinar del Rio) à Cuba. U.S.N.M. Helm. Coll. no 71795. Nous remercions le Professeur J. R. Lichtenfels de l'Institut de Parasitologie animale de Beltsville (Maryland) qui nous a permis d'étudier ces spécimens.

\section{- Redescription :}

1. Structures céphaliques : dans les deux sexes trois pseudo-lèvres en position interradiale bordent l'ouverture buccale ( $f i g .1 C$ et $3 \mathrm{~A}$ ); les terminaisons nerveuses étroitement regroupées latéralement ( fig. $1 \mathrm{D}$ ) bordent les deux pseudo-lèvres latéro dorsales ; chaque pseudo-lèvre est renforcée en profondeur par un interlabia qui vient s'intercaler entre les dents œsophagiennes adjacentes (fig. $1 E$ et $3 \mathrm{~A}$ ); vésicule céphalique bien développée. Chez la femelle l'extrémité céphalique est relativement plus large, et les pseudo-lèvres sont plus développées (fig. 1 C).

2 - Organes génitaux femelles : l'appareil génital présente la même disposition générale décrite dans le genre par Quentin (1969), vulve relativement postérieure, trompe utérine faisant un coude vers l'avant (fig. $1 \mathrm{~A}$ ); une jeune femelle, non gravide, avait conservé la selle de copulation sécrétée par le mâle au cours de l'accouplement ( fig. $1 \mathrm{H}$ et I) ; œuf asymétrique, non operculé ( fig. $1 \mathrm{~B}$ ).

3 - Organes génitaux mâles :

a) area rugosa, deux mamelons ventraux sont suivis par plusieurs rangées de plis pectinés peu marqués, occupant la partie médiane de chaque interstrie ventrale

FIg. I. - Helminthoxys tiflophila (Vigueras, r943) 우

A, vue latérale droite ; B, œuf ; C, tête, vue apicale ; D, id., détail des terminaisons nerveuses céphaliques, en vue latérale; $\mathrm{E}$, id., coupe optique en vue apicale, passant par la base des pseudolèvres; F, id., coupe optique en vue ventrale, passant par les amphides; G, coupe transversale du corps, passant par la vulve ; selle de copulation : $\mathrm{H}$, côté $q$ et $\mathrm{I}$, côté $\widehat{\sigma}$.

Valeur de l'échelle: A, I $650 \mu \mathrm{m}$; B et E, roo $\mu \mathrm{m}$; C et F, $250 \mu \mathrm{m} ; \mathrm{D}, 50 \mu \mathrm{m} ; \mathrm{G}, \mathrm{H}$ et I, $500 \mu \mathrm{m}$. 


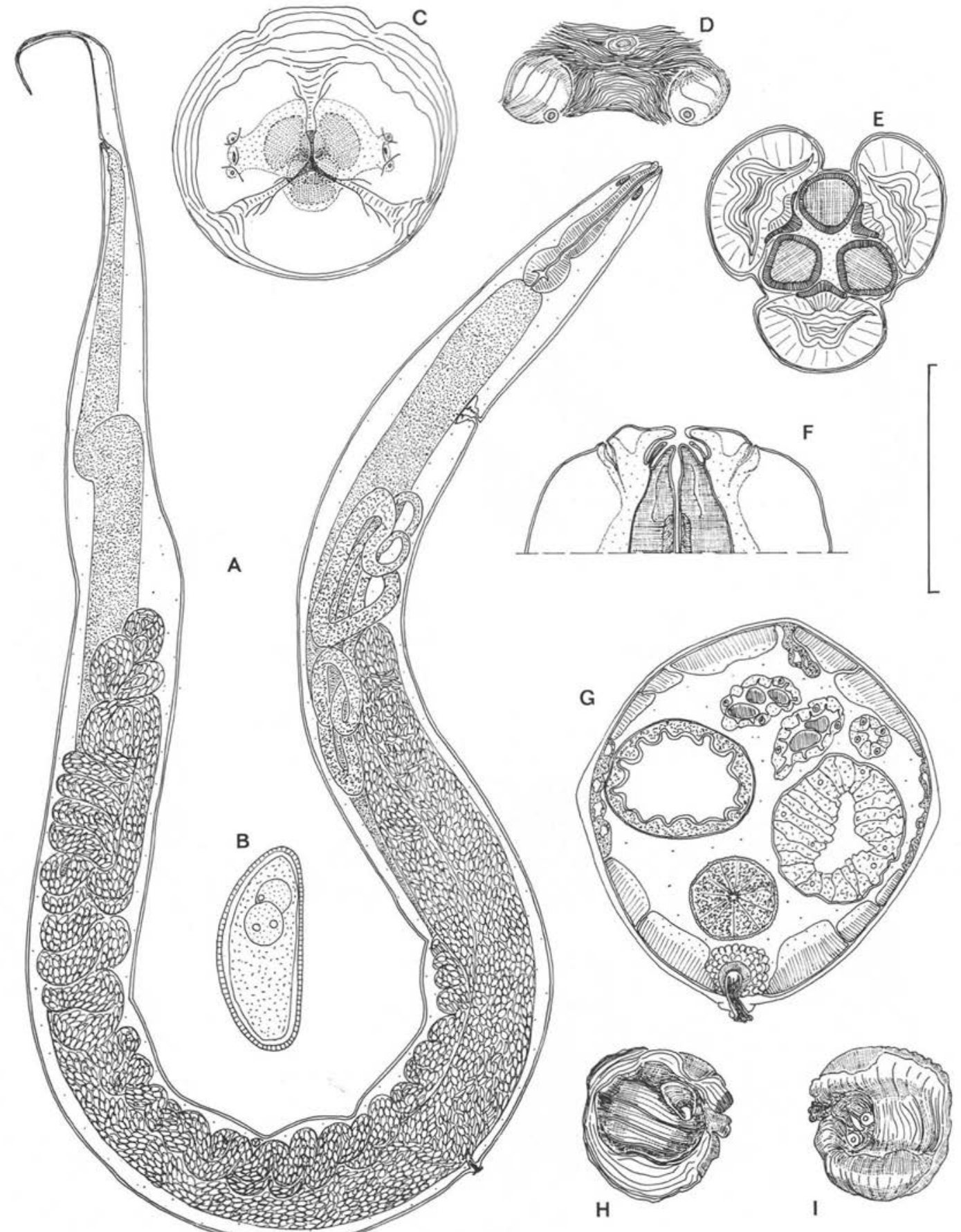

FIG. I. 

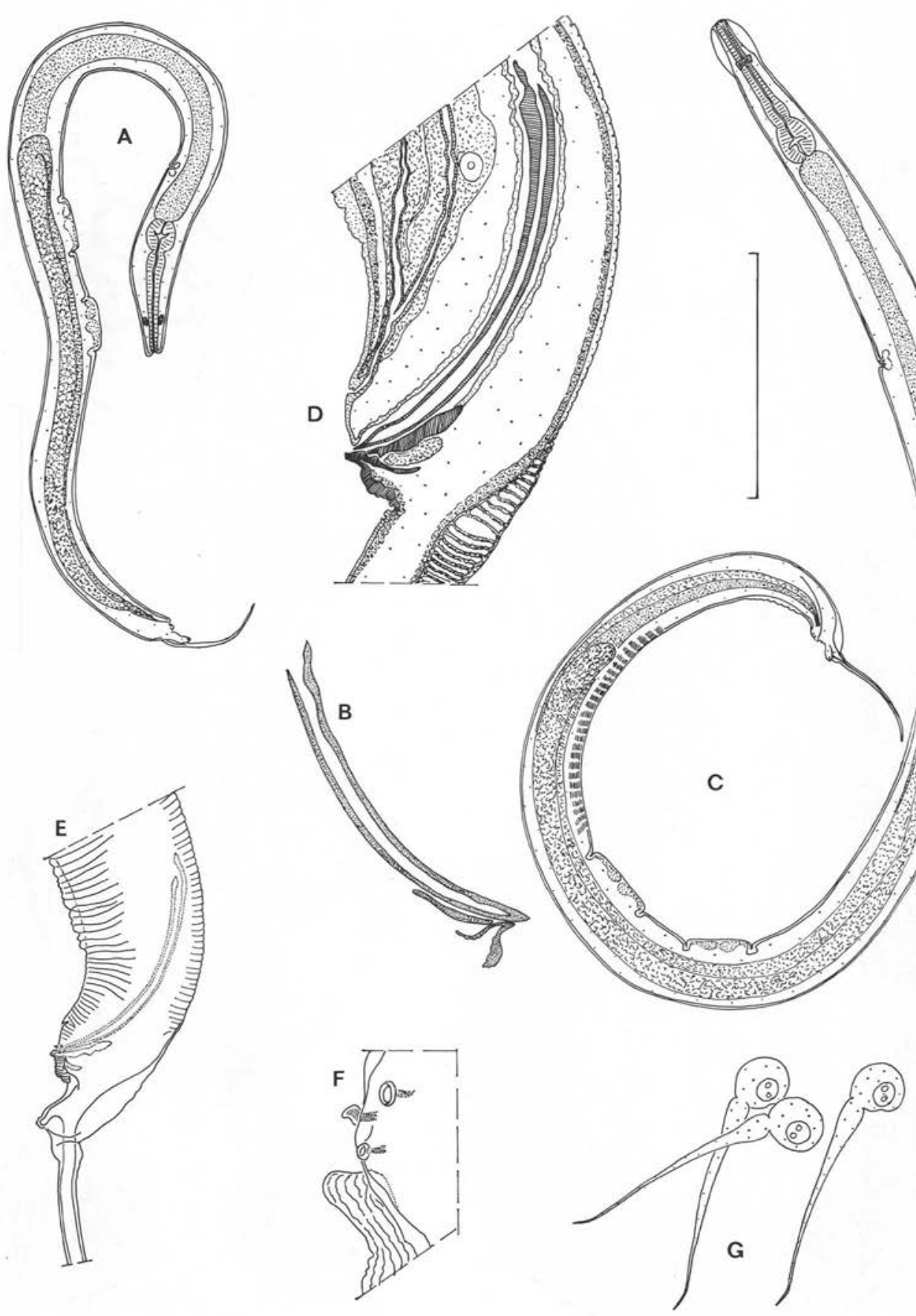

FIG. 2. 
( fig. 2 C) ; chaque mamelon porte en surface cinq sillons parallèles, obliques par rapport au plan sagittal (fig. $3 F$ ) ; au fort grossissement chaque sillon paraît partiellement recouvert par une croûte brunâtre d'aspect analogue à celui des sécrétions cloacales du mâle (selle et bouchon de copulation : figures $1 \mathrm{~A}, \mathrm{G}, \mathrm{H}$ et $\mathrm{I}$ ) ; une coupe transversale du corps (fig. $3 \mathrm{D}$ et $E$ ) révèle une modification des tissus du champ ventral : une formation glandulaire est reliée aux sillons par un canal dont la lumière débouche sous la cuticule de chacun des mamelons (fig. $3 \mathrm{E}$ ) ; l'une des cellules musculaires ventrale médiane est hypertrophiée et l'orientation de ses myofibrilles est modifiée au voisinage du champs ventral (fig. $3 \mathrm{D}$ et E).

b) bourse caudale (figures $2 D, E$ et $F$ et $3 B$ et $C$ ) ;

c) organes copulateurs : spicule bien chitinisé; gubernaculum composé d'un corpus oblong peu développé et d'un crochet accessoire important dont la partie ventrale forme un relief massif et chagriné en surface, qui borde postérieurement l'ouverture cloacale ( fig. $2 B, D$ et $E$ et $3 B$ et $C$ ).

- Mensuration (tableau I).

TABleau I. - Helminthoxys tiflophila (Vigueras, 1943)

\begin{tabular}{|c|c|c|c|}
\hline $\begin{array}{l}\text { Mensurations } \\
\text { en } \mu \mathrm{m}\end{array}$ & 우 & fig. $2 \mathrm{~A}$ & fig. ${ }^{\hat{\sigma}}$ \\
\hline Écart des pores amphidiaux & 170 & - & 140 \\
\hline Longueur du corps & 19200 & 7700 & 13400 \\
\hline Largeur du corps & 800 & 300 & 410 \\
\hline Longueur de l'œsophage & 1250 & 900 & 1100 \\
\hline Diamètre du bulbe & 350 & 250 & 300 \\
\hline $\begin{array}{l}\text { Distance apex : } \\
\text { anneau nerveux } \\
\text { pore excréteur } \\
\text { vulve }\end{array}$ & $\begin{array}{rl} & 200 \\
2 & 250 \\
8 & 000\end{array}$ & $\begin{array}{r}200 \\
1250 \\
-\end{array}$ & $\begin{array}{r}300 \\
2500 \\
-\end{array}$ \\
\hline Longueur du spicule & - & 390 & 460 \\
\hline Longueur du gubernaculum & - & 115 & 130 \\
\hline Longueur de la queue & 1600 & 750 & 950 \\
\hline Longueur de la pointe caudale & - & 650 & 800 \\
\hline Dimensions des œufs & $90 \times 40$ & - & - \\
\hline
\end{tabular}

FIG. 2. - Helminthoxys tiflophila (Vigueras, 1943) $\widehat{\jmath}$

Jeune adulte: A, vue latérale droite; B, organes copulateurs, détail ; adulte mûr: C, vue latérale gauche; $\mathrm{D}$, bourse caudale, coupe optique passant par le plan saggital, en vue latérale gauche ; E, id., vue superficielle; F, terminaisons nerveuses latérales au cloaque (détail de la précédente) ; G, spermatozoïdes.

Valeur de l'échelle : A et C, I $650 \mu \mathrm{m}$; B et D, $250 \mu \mathrm{m} ; \mathrm{E}, 500 \mu \mathrm{m}$; F et G, $50 \mu \mathrm{m}$. 

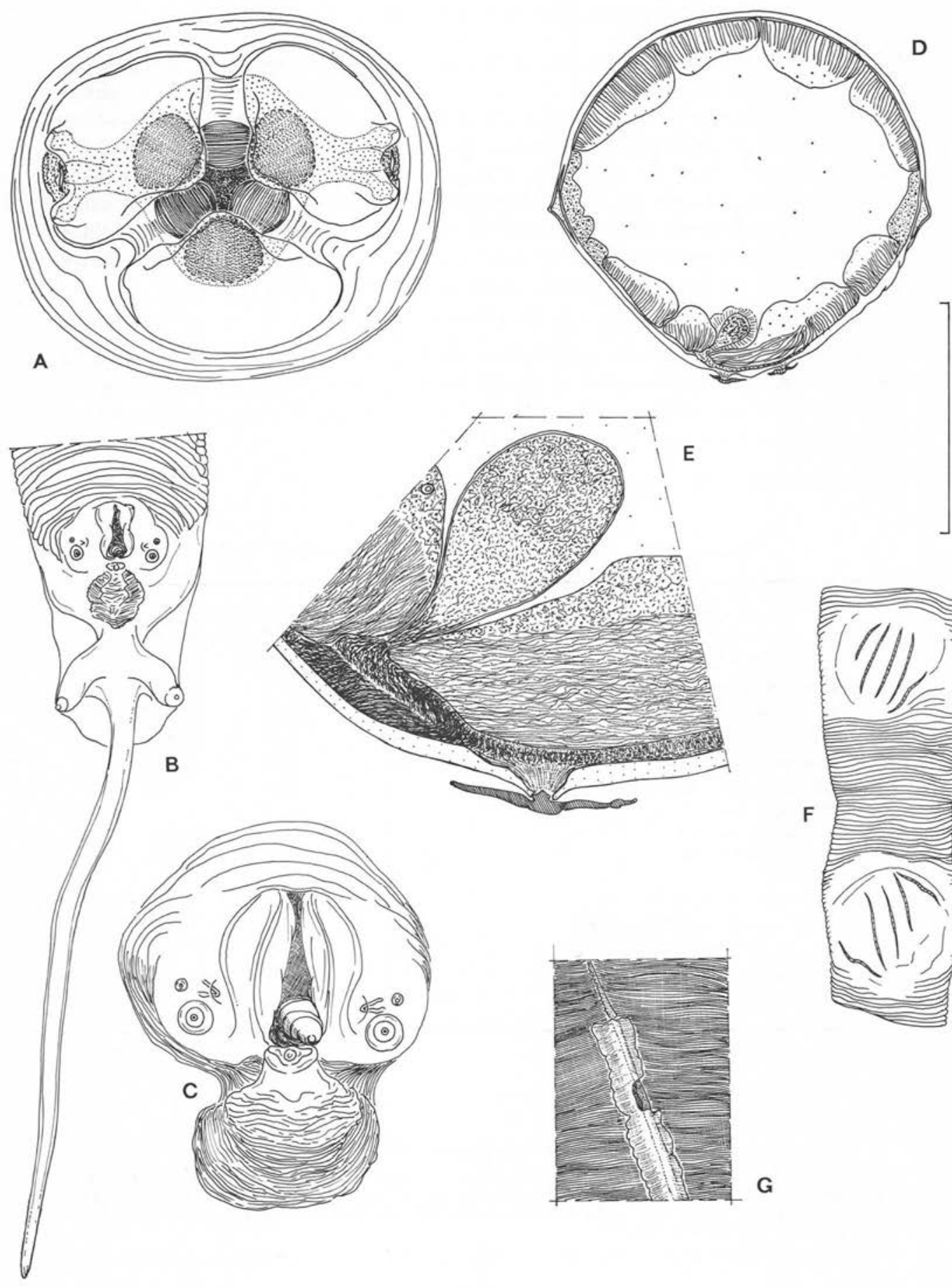


\section{Discussion}

Les caractères et les mensurations de nos spécimens correspondent à ceux d'Helminthoxys tiflophila décrits par Vigueras (1943) et par Barus (1972) chez le même hôte et dans la même localisation géographique et nous les identifions à cette espèce.

\section{Analyse morphologique du genre Helminthoxys}

- Bourse caudale (caractères 1 et 2).

Chez $H$. tiflophila comme chez toutes les autres espèces du genre Helminthoxys dont les descriptions sont suffisantes, la bourse caudale est caractérisée par la présence :

- d'une seule paire de papilles pericloacales fortement pédonculées ; ces papilles sont postcloacales et encadrent la pointe caudale bien développée (caractère 1).

- de trois paires de papilles latérales au cloaque (caractère 2).

Le caractère 1 est commun à tous les Oxyuridae parasites de Rongeurs ou de Lagomorphes à l'exception du genre Citellina Prendel, 1948 parasite de Sciuridés holarctiques. Le caractère 2 n'est rencontré que dans quatre genres : Protozoophaga Travassos, 1923 - Wellcomia Sambon, 1907 (sensu Hugot, 1982a) - Helminthoxys Freitas, Lent et Almeida, 1937 - Octodonthoxys Quentin, Courtin et Fontecilla, 1975, tous parasites de Rongeurs Caviomorphes ou Phiomorphes (= Hystricognathes sensu Chaline et Mein, 1979).

- Ornementation ventrale (caractères 3,4 et 5):

- plis pectinés (caractère 3) : les séries de plis pectinés rencontrées entre le deuxième mamelon et le cloaque chez H. tiflophila (fig. 2) ont été décrites dans la même disposition chez la plupart des autres espèces du genre et également dans le genre Octodonthoxys (voir Quentin, 1973 et 1975). Dans un travail récent nous avons montré que ce type d'ornementation doit être considéré comme primitif : dans les genres Protozoophaga et Wellcomia ces plis pectinés se transforment et donnent naissance à des rangées parallèles de crêtes cuticulaires longitudinales (voir DiazUngria et Quentin, 1977 et Hugot, 1982a).

FIG. 3. - Helminthoxys tiflophila (Vigueras, 1943) $\hat{\sigma}$

A, tête, vue apicale ; B, bourse caudale, vue ventrale ; C, id., détail de l'ouverture cloacale ; $\mathrm{D}$, coupe transversale du corps au niveau du mamelon antérieur ; E, id., détail du champ ventral ; $\mathrm{F}$, mamelons, vue ventrale; $\mathrm{G}$, id., détail d'un sillon.

Valeur de l'échelle : A et C, $100 \mu \mathrm{m}$; B et D, $250 \mu \mathrm{m}$; E et G, $50 \mu \mathrm{m} ; \mathrm{F}, 825 \mu \mathrm{m}$. 
- mamelons (caractères 4 et 5) : les mamelons cuticulaires ventraux apparaissent dans toutes les lignées d'Oxyuridae parasites de Rongeurs et de Lagomorphes qui possèdent en commun le caractère 1 . Ces mamelons ont une anatomie variable selon les taxa et l'on peut rattacher chaque type particulier à une ornementation plus simple, rencontrée dans le même taxon chez des espèces plus primitives (voir Hugot 1981, 1982a et Hugot, sous presse a et b). Chez les Oxyuridae de Rongeurs les mamelons sont donc apparus plusieurs fois, par convergence, dans différentes lignées. L'étude détaillée de ces mamelons montre, chez la plupart d'entre eux, l'existence de reliefs cuticulaires particuliers augmentant l'adhérence du mamelon sur la cuticule de la femelle pendant l'accouplement (voir Dick et Wright, 1974 - Hugot, 1982a et Hugot sous presse a et b). Le mamelon décrit chez $H$. tiflophila présente donc la singularité suivante : la coaptation étroite avec la cuticule femelle n'y est pas assurée par " accrochage » (caractère 4), mais par "collage » (caractère 5), la sécrétion adhésive du champ ventral jouant un rôle analogue à celui des sécrétions du cloaque, dont la coagulation permet la formation de la selle de copulation (voir Seurat, 1920). Des mamelons de type 5 ont été décrits dans toutes les espèces des genres Helminthoxys et Octodonthoxys. Chez Wellcomia le mamelon est du type 4 ; chez Protozoophaga il n'y a pas de mamelon.

\section{- Gubernaculum (caractère 6 et 7).}

La présence d'un crochet accessoire sur le gubernaculum est un caractère évolué qui apparaît dans différentes lignées. Il a la forme d'un simple épaississement de la lèvre postérieure du cloaque (caractère 6) ou bien, comme chez $H$. tiflophila (fig. 2) l'aspect général en vue latérale d'un fer à cheval (caractère 7). Dans les genres Helminthoxys et Octodonthoxys le gubernaculum est toujours du type 7, dans le genre Wellcomia toujours du type 6, dans le genre Protozoophaga le crochet accessoire est absent.

- Spermathèque (caractère 8).

Chez tous les Oxyuridae de Rongeurs possédant en commun le caractère 2 et dont l'appareil génital femelle est suffisamment connu on rencontre une spermathèque d'un type particulier, dont la description détaillée a été donnée dans un travail précédent (Hugot, 1982a) : à la jonction de l'utérus et de l'oviducte une formation sub-sphérique renferme les spermatozoïdes venus s'y accumuler après l'insémination; autours de ce réservoir l'oviducte se replie en épingle à cheveux.

- Formations buccales (caractères 9, 10 et 11)

Chez Helminthoxys et Octodonthoxys la capsule buccale renferme des dents œsophagiennes et des interlabia simples ( fig. 1) ; ces formations s'opposent à celles rencontrées chez Protozoophaga et Wellcomia dont les dents œsophagiennes portent des reliefs apicaux (caractère 9), eux-mêmes intriqués avec des interlabia complexes (caractère 10) (voir Diaz-Ungria et Quentin, 1977 et Hugot, 1982a). Chez Protozoophaga les reliefs dentaires apicaux ont la forme d'une spatule (caractère 11). 


\section{Interprétation cladistique}

En récapitulant, les onze caractères que nous venons d'énumérer et les taxa correspondants on obtient le tableau II. Le signe + signifie qu'un taxon possède le caractère correspondant dans son état apomorphe (évolué), le signe - qu'il le possède dans son état plésiomorphe (primitif), le signe • que ce caractère est indifférent pour le taxon considéré : c'est-à-dire qu'on peut l'y rencontrer sous une forme analogue, mais non homologue.

TABleau II. - (Voir explication dans le texte).

\begin{tabular}{|c|c|c|c|c|c|c|c|c|c|c|c|}
\hline & 1 & 2 & 3 & 4 & 5 & 6 & 7 & 8 & 9 & 10 & 11 \\
\hline Octodonthoxys & + & - & - & - & + & - & + & + & - & - & . \\
\hline Helminthoxys & + & - & - & - & + & - & + & + & 一 & - & . \\
\hline Wellcomia & + & - & + & + & - & + & - & + & + & + & - \\
\hline Protozoophaga & + & 一 & + & - & - & - & - & + & + & + & + \\
\hline $\begin{array}{l}\text { Oxyuridae de Rongeurs } \\
\text { Sciurognathes et de } \\
\text { Lagomorphes }\end{array}$ & + & + & . & . & . & . & . & - & . & . & . \\
\hline $\begin{array}{l}\text { Autres Oxyuridae } \\
+ \text { Citellina }\end{array}$ & - & . & . & . & . & . & . & . & . & . & . \\
\hline
\end{tabular}

- Analyse des caractères

Caractère 1. Chez les Oxyures parasites de Vertébrés, le nombre des papilles caudales du mâle tend à se réduire et leurs pédoncules tendent à se raccourcir (Petter et Quentin, 1976) ; la présence d'une seule paire de papilles péricloacales pédonculées chez certains Oxyuridae est par conséquent une apomorphie puisque tous les autres Oxyuridae en possèdent au moins deux paires.

Caractère 2. Pour les mêmes raisons la présence de deux paires seulement de papilles latérales au cloaque est une apomorphie, la présence de trois paires de papilles est une plésiomorphie.

Caractère 3. La présence de plis pectinés est la forme plésiomorphe d'une ornementation cuticulaire dont la forme apomorphe est constituée par des rangées de crêtes longitudinales (voir Hugot, 1982a).

Caractères 4 et 5. Le mamelon à " accrochage " (caractère 4) ou à " collage " (caractère 5) sont apomorphes, l'absence de l'une ou l'autre de ces formes est plésiomorphe. Caractères 6 et 7. La présence d'un crochet accessoire est un caractère apomorphe. Caractère 8. La spermathèque particulière rencontrée chez les Oxyuridae de Rongeurs Hystricognathes nous paraît plus différenciée que celles qui ont été décrites chez 


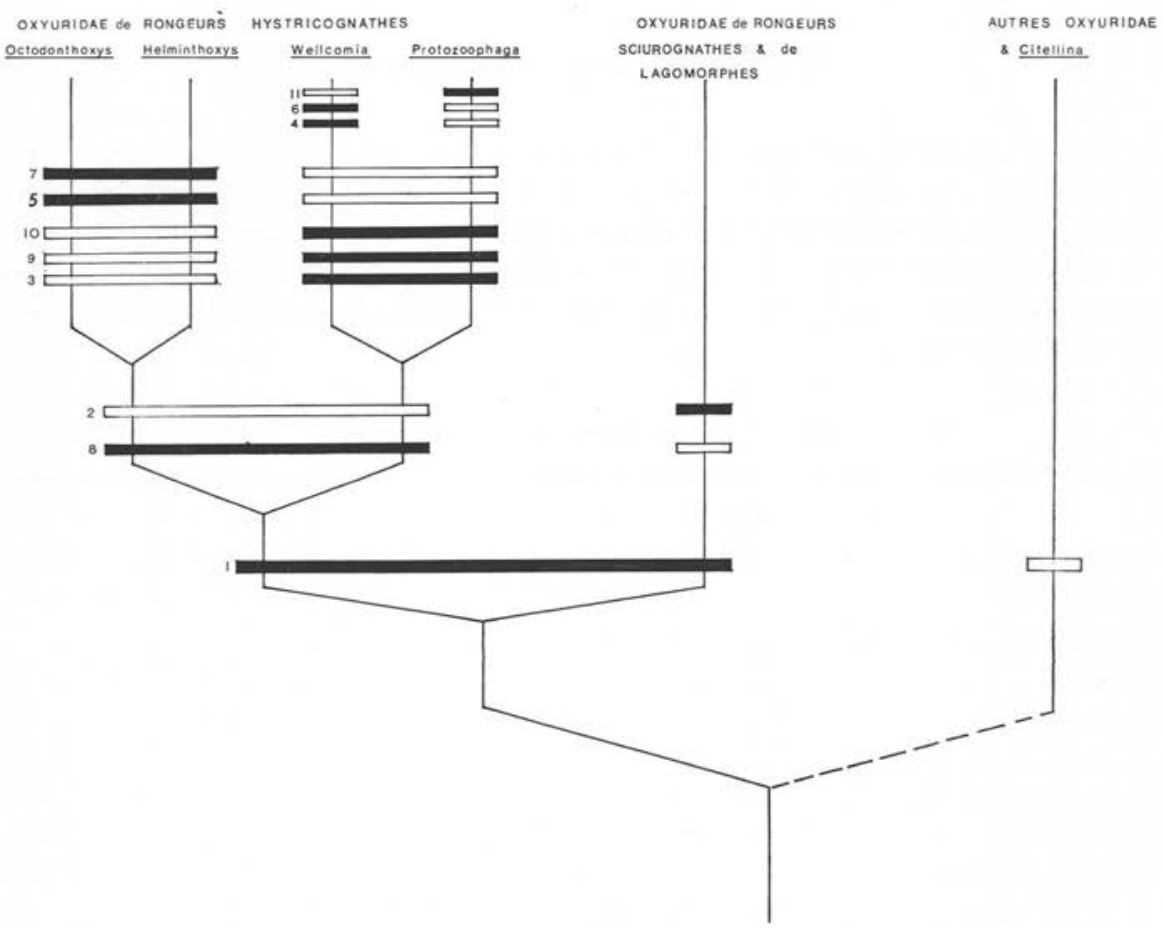

Frg. 4. - Relations phylogénique des Oxyuridae Parasites de Rongeurs.

Les chiffres se réfèrent aux caractères étudiés dans le texte. Les rectangles blancs indiquent la présence de la forme plésiomorphe et les rectangles noirs de la forme apomorphe d'un caractére.

d'autres Oxyuridae de Rongeurs et qui sont de simples dilatations ampulaires de l'oviducte. Nous choisissons par conséquent d'interpréter la présence de ce caractère comme une apomorphie.

Caractère 9, 10 et 11 . Nous considérons la présence de formations buccales plus complexes comme des apomorphies et nous interprétons les reliefs dentaires apicaux en spatule rencontrés chez Protozoophaga comme une autapomorphie.

- Recherche des taxons frères.

Le tableau II permet de construire le cladogramme figuré en 4, dont on peut faire l'analyse suivante :

1 - le groupe Octodonthoxys - Helminthoxys - Wellcomia - Protozoophaga peut être considéré comme le taxon frère de l'ensemble des autres Oxyuridae de Rongeurs et de Lagomorphes, moins Citellina, pour les caractères 1, 2 et 8 .

2 - à l'intérieur de ce groupe, Octodonthoxys - Helminthoxys est le taxon frère de Wellcomia - Protozoophaga, pour les caractères 3, 5, 7, 9 et 10 .

3 - Wellcomia est le taxon frère de Protozoophaga, pour les caractères 3,9 et 10. 
4 - les caractères 4 et 6 sont autapomorphes pour Wellcomia.

5 - le caractère 11 est autapomorphe pour Protozoophaga.

6 - les caractères que nous avons choisis ne permettent pas de distinguer Octodonthoxys et Helminthoxys.

\section{Conclusion}

A l'exception du genre Citellina, qui appartient à un autre groupe, les Oxyuridae parasites de Rongeurs et de Lagomorphes constituent un ensemble monophylétique. A l'intérieur de cet ensemble le genre Helminthoxys et les genres voisins Octodonthoxys, Wellcomia et Protozoophaga, tous parasites de Rongeurs Hystricognathes, s'opposent aux Oxyuridae parasites de Rongeurs Sciurognathes (sensu Chaline et Mein, 1979) et de Lagomorphes.

\section{BIBLIOGRAPHIE}

BARUS, V. : Remarks ou the Cuban species of the genus Helminthoxys (Nematoda, Syphaciidae). Folia Par., 1972, 19, 105-III.

Chaline J., Mein P. : Les Rongeurs et l'Évolution, Doin, Paris, 1979, 236 p.

DiAz-Ungria G., Quentin J.-C. : Morphologie et position systématique de l'Oxyure Protozoophaga obesa (Diesing, 1851) Travassos, 1923. Excerta Parasitologica en Memoria del Doctor Eduardo Caballero y Caballero, Universidad Nacional de Mexico, Instituto de Biologia, Publicaciones especiales, $1977,4,423-428$

Dick T. A., WRIGHT K. A. : The ultrastructure of the cuticle of the nematode Syphacia obvelata (Rudolphi, 1802). III. Cuticle associated with the male reproductive structures. Can. J. Zool., 1974, s2, 179-182.

Freitas Texeira J. F., Lent H., Almeida J. L. : Pequena contribuiçao ao estudo da fauna helminthologica de Argentina (Nematoda). Mem. Inst. Oswaldo Cruz, 1937, 32, 195-209.

Hugot J. P. : Les Syphaciinae (Oxyuridae) parasites de Sciuridés. Évolution des genres Syphatineria et Syphabulea. Mem. Mus. Nat. Hist. Nat., Paris, A Zool., 1981, 121, I-64.

Hugot J. P. : Sur le genre Wellcomia (Oxyuridae, Nematoda), parasite de Rongeurs archaïques. Bull. Mus. Nat. Hist. Nat., Paris, $4^{\mathrm{e}}$ sér, 1982 a, 4 , section A (I-2), 25-48.

Hugot J. P. : Zenkoxyuris quentini (Nematoda) : Un nouvel oxyure d'Anomalure. Bull. Mus. Nat. Hist. Nat., Paris, $4^{\mathrm{e}}$ sér., 1982 b, 4 , section A (I-2), 49-59.

Hugot J. P. : Deux Oxyures parasites du Rat des Rochers sud-africain. Bull. Mus. Nat. Hist. Nat., Paris $4^{\mathrm{e}}$ sér., $\mathrm{r} 983$, , , section $\mathrm{A}(\mathrm{I})$, (sous presse a).

Hugot J. P. : Redescription de Zenkoxyuris mabokensis (Oxyuridae, Nematoda) parasite de Zenkerella insignis (Anomaluridae, Rongeur) Systematic Parasitology (sous presse b).

Petter A., Quentin J. C. : Keys to genera of the Oxyuroidea. C. I. H. Keys to the Nematode parasites of Vertebrates. IV - Commonwealth Institute of Helminthology, the White House, 103 St Peter's Street, St Albans, Herts, England, 1976, 29 p.

Quentrin, J. C. : Les Onyurinae de Rongeurs. Bull. Mus. Nat. Hist. Nat., Paris, $3^{\mathrm{e}}$ sér., I973, Zool. 112 , 1045-1096.

Quentin J.-C., Courtin Lyon S., Fontecilla Gallardo J. : Octodonthoxys gigantea n. gen., n. sp. nuevo nematodo Oxyurinae, parasito de un rodeor caviomorpho de Chile. Boln. Chil. Parasit., 1975, 30, 21-25.

Sambon L. W. : Descriptions of some new species of animal parasites. Proc. zool. Soc. London, $1907, x, 282-283$.

Seurat L. G. : Histoire Naturelle des Nématodes de la Berberie. Première partie : morphologie, développement, éthologie et affinités des Nématodes. Alger Imprimeries, S. Stanuel, I920, p. $22 \mathrm{I}$-VI.

Travassos L. : Informaçoes sobre a fauna helminthologica de Mato Grosso. Folha Med., 1923, 4, 35-36.

Vigueras, I. P. : Un genero y cinco especias nuevas de helminthos cubanos. Univ. Habana, 1943 , 8. $315-356$. 